\title{
Multi-ETS carbon prices forecasting based on EMD-SVM model
}

\author{
Xiaoli Yang ${ }^{1}$, Shanglei Chai ${ }^{1, *}$, Huizheng Chen ${ }^{1}$ and Zixuan Zhang ${ }^{1}$ \\ ${ }^{1}$ Business School, Shandong Normal University, 88 Wenhua Road, Jinan 250014, China
}

\begin{abstract}
With the widespread attention of governments around the world on climate issues, carbon pricingrelated policies have been gradually adopted by countries to deal with climate change. Among these policy tools, the carbon emissions trading system (ETS) is the most widely used. Carbon price plays a crucial role in this trading system, not only determining the trading activity, but also affecting the market stability. Therefore, carbon price prediction is so significant that we are motivated to study it. However, carbon price presents complex nonlinear dynamic characteristics, which makes some existing methods inaccurate. To address it, this paper combines empirical mode decomposition (EMD) and support vector machine (SVM) to predict carbon prices. The original carbon prices are signal-decomposed by using EMD and the decomposed signal is predicted by using SVM. Based on the EMD-SVM model, this paper conducts empirical analysis on the carbon prices of multi-ETS, including European Union ETS and China ETS pilots. The results of analysis show that the EMD-SVM model has better overall forecasting ability, and carbon prices forecasting performance of China ETS pilots is better than that of the EU ETS, while the short-term forecasting results of the model show the opposite conclusion. The proposed EMD-SVM model is advisable in carbon prices forecasting for market participants and regulatory authorities of multi-ETS.
\end{abstract}

\section{Introduction}

Since the industrial revolution, global climate change caused by excessive carbon emissions has had a serious impact on the environment and human life, and experience has shown that it is difficult to achieve the desired goal by government compulsory requirements or voluntary reduction of economic entities. On February 16, 2005, the "Kyoto Protocol" came into effect, and countries established carbon emission trading systems (ETS) successively, so that carbon emission rights have commodity attributes and can be traded in the market. China's united carbon emissions trading market was officially established on December 19, 2017, and is still in its initial stage.

To a certain extent, predicting carbon prices can get rid of China's current passive situation in the international carbon market, improve the risk precaution ability of the enterprise while reducing the loss of its carbon product prices, so the research of this paper has important theoretical and practical significance.

Due to the different maturity of the carbon market around the world, carbon prices are affected by different factors, showing different volatility characteristics, so research models for predicting carbon prices are not the same for different markets. However, models on carbon prices prediction are basically divided into three categories: traditional econometric models, machine learning models and hybrid models. ${ }^{[1]}$ The traditional econometric models mainly includes generalized autoregressive conditional heteroskedasticity model $(\mathrm{GARCH})^{[2]}$, autoregressive model (AR) and autoregressive moving average model (ARMA) $)^{[3]}$, Autoregressive Integrated Moving Average (ARIMA) models, etc. For example, Byun et al. (2013) ${ }^{[4]}$ studied the volatility prediction ability of GARCH-type models, and found that the prediction results of GARCH-type models are better than AR model.

However, carbon prices time series has its own nonlinear and non-stationary characteristics ${ }^{[5]}$, which makes traditional prediction models in poor performance. As a result, researchers introduced machine learning models to carbon prices prediction. It was considered to have strong promotion, high-speed computing features ${ }^{[6]}$, such as k-Nearest Neighbour (KNN), back propagation neural network (BPNN), least squares support vector (LSSVM) ${ }^{[7]}$. However, the use of a single machine learning model does not allow high-precision prediction of complex nonlinear carbon prices predictions. Zhu and Wei (2011) ${ }^{[7]}$ used the hybrid model of GMDH-PSOLSSVM to predict the carbon prices of the EU ETS. The results showed that the hybrid model was better than single model.

The above literature rarely compared the prediction effects of different carbon emission systems with a hybrid model containing machine learning model, and studied the applicability of the models under different trading systems. This paper used EMD-SVM hybrid model to forecast carbon prices of the EU ETS and China ETS pilots, in order to compare the prediction effects of the hybrid models in these markets. The empirical mode decomposition (EMD) proposed by Huang et al. (1998) ${ }^{[8]}$ was widely used in multiple time series fields ${ }^{[9-12]}$.

\footnotetext{
* Corresponding author: chaishanglei@sdnu.edu.cn
} 
Based on the above analysis, the carbon prices have a highly complex nonlinear characteristic in the carbon markets around the world. When forecasting carbon prices, this paper uses EMD-SVM hybrid model to predict the carbon prices and observed the prediction performance of the three carbon markets. So that we can find a more suitable carbon prices forecasting market for this model.

\section{Methodology}

\subsection{Empirical Mode Decomposition (EMD)}

Empirical Mode Decomposition (EMD) is an adaptive signal decomposition method for nonlinear, nonstationary signals proposed by Huang et al. (1998). ${ }^{[8]}$ It can decompose the fluctuations of different time scales in the signal into several Intrinsic Mode Functions (IMFs) with different frequencies and one residue. And the residue represents the overall trend of original signal. The specific decomposition method of EMD is as follows. ${ }^{[1]}$

Step 1: The EMD model finds the extreme points of the original sequence $x(t)$, and uses the cubic spline function to fit the extreme points respectively to form the upper and lower envelopes. Then the model averages the two envelopes and the average envelope $m_{1}(t)$ is obtained.

Step 2: Subtract the original sequence $x(t)$ from the average $m_{1}(t)$ obtained in Step 1, and yield a new sequence $h_{1}(t)$ :

$$
h_{1}(t)=x(t)-m_{1}(t)
$$

Step 3: Check $h_{1}(t)$. When $h_{1}(t)$ meets the definition of IMF, $h_{1}(t)$ is defined as an IMF $c_{1}(t)$. Otherwise, let

$$
x(t)=h_{1}(t)
$$

return to the Step 1, and cannot repeat unless $h_{1}(t)$ meets the conditions.

Step 4: Subtract the first IMF $c_{1}(t)$ from the original sequence $x(t)$ and obtain the residue $r_{1}(t)$,

$$
r_{1}(t)=x(t)-c_{1}(t)
$$

Step 5: Perform the steps 1-4 only when the termination criterion is met.

Finally, we obtain

$$
x(t)=\sum_{j=1}^{n-1} c_{1}(t)+r_{n}(t)
$$

\subsection{Support Vector Machine (SVM)}

SVM is a machine learning model proposed by Cortes and Vapnik (1995) [13], which follows the principle of structural risk minimization rather than empirical risk minimization. And it can be accurately implemented for small-sample high-dimensional nonlinear systems, which has a good generalization ability. ${ }^{[14]}$
To put it simply, nonlinear separable problem is converted into linear separable problem and the real space is transformed into a feature space. The regression function of SVM in high dimensional feature space is:

$$
f(x)=w \cdot \varphi(x)+b
$$

where $\varphi(x)$ is a nonlinear mapping function, $x \in$ $R^{m}, w$ is weighted vector and $b$ is biased vector.

\section{Empirical analysis}

\subsection{Data description}

At present, China's national unified carbon market is still in the construction stage; there is no national trading activity, only transaction data of eight pilots. On June 18, 2013, Shenzhen pilot firstly launched carbon emissions trading in China, then Hubei pilot began trading in the following year. At present, Hubei has become the most significant trading volume among the eight pilots, so this paper selects Hubei and Shenzhen two pilot cities. In order to ensure the data comparability and time consistency, the three markets selected data from May 1, 2014, to March 29, 2018, as shown in Fig. 1. This paper divided the total sample into training samples and test samples in a ratio of $8: 2$.

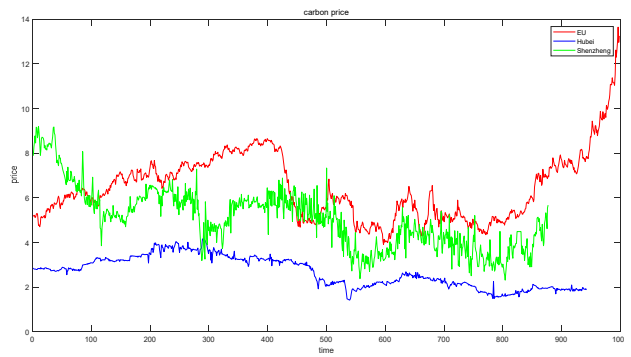

Fig. 1. The daily carbon prices of the study areas.

\subsection{Empirical results}

\subsubsection{EMD.}

For the adaptive algorithm EMD, it can automatically decompose different numbers of IMF according to the data characteristics, without pre-setting the number of IMF. And the final EMD results of carbon prices were shown in Fig. 2. As shown in Fig. 2(a), the frequency of change of IMF1 is high and the fluctuation is significant, which reflects the noise information of the original signal. The frequency of change of IMF2-IMF6 and the residue is low, including the periodicity and trend information of the original signal.

Comparing the high-frequency signals of the three markets, it is found that the volatility of Fig. 2(a) is more significant than that of Fig. 2(b) and (c). We believe that this phenomenon may be caused by the following factors. The EU ETS has developed rapidly from 2014 to 2018, and carbon prices have also increased. But it has been affected by some exogenous events, such as compliance 
events $^{[15]}$, carbon emissions information disclosure ${ }^{[16,17]}$, which would result in higher fluctuations in carbon prices at higher prices.

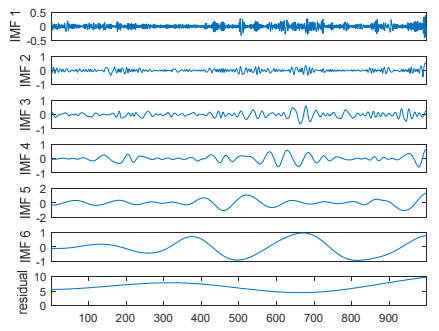

(a) EU ETS

(b)Shenzhen
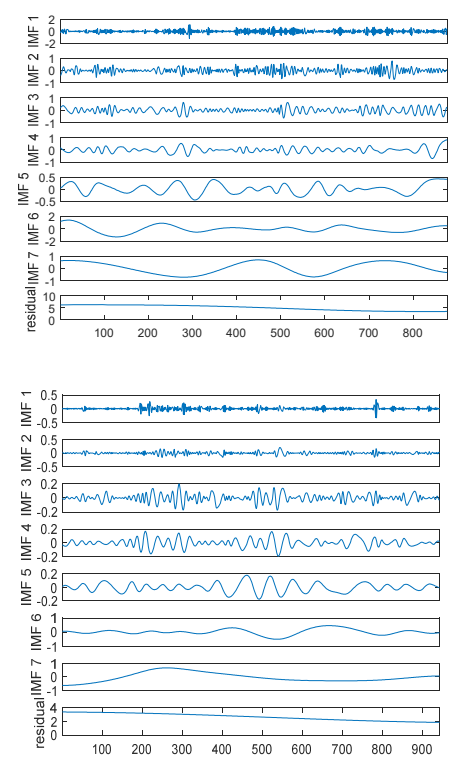

(c)Hubei

Fig. 2. Carbon prices decomposition.

\subsubsection{SVM forecast.}

In view of the fact that the radial basis kernel function has fewer parameters and better performance than other kernel functions, the radial basis kernel function is used as the kernel function of SVM. The number of input variables and the coefficient of the insensitive loss function is obtained by cross-validation. This paper normalized the eigenvalues, then returned the results to the real value after deriving the results. The comparison and errors of the forecasting results of the three markets are shown in Figs 3-5.

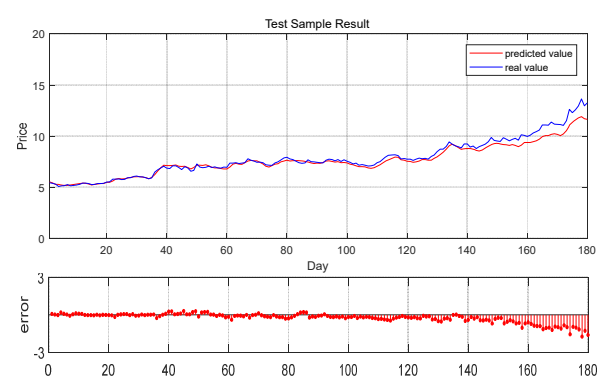

Fig. 3. EU ETS carbon prices forecasting.
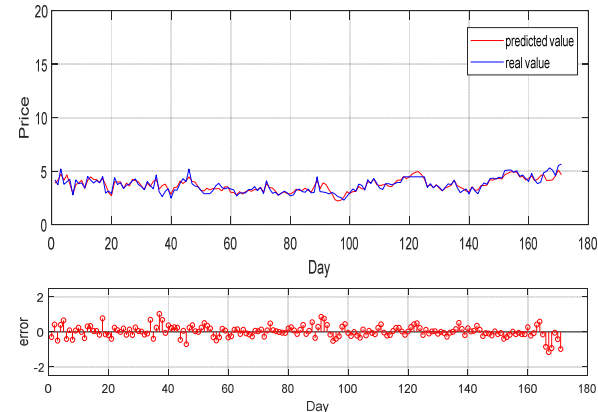

Fig. 4. Shenzhen carbon prices forecasting.

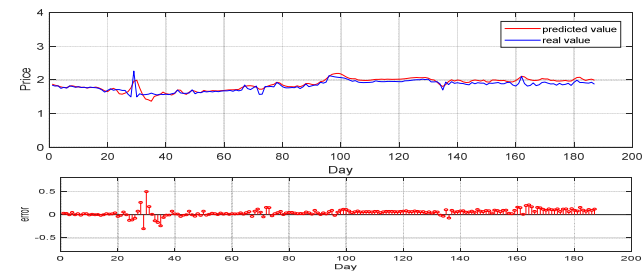

Fig. 5. Hubei carbon prices forecasting.

Figs 3-5 indicate that the overall trend of the prediction made by the EMD-SVM model is almost the same as the real value, which is consistent with the trend of carbon prices ${ }^{[18]}$, but the prediction of the specific prices is still not very accurate. Fig. 3 shows the prediction error of the EU carbon prices, the error is still relatively small in the short term, and not significantly fluctuated. But in the long term, as the time increases, the error increases gradually. As shown in Figs 4 and 5, when predicting carbon prices of the China ETS pilots, the shortterm forecast has a smaller error and better performance than the long-term forecast. Unlike Figs 3-5 shows that the error fluctuations in the short-term are large, and the stability of the prediction accuracy is poor. Fig. 5 shows a significant fluctuation in one place. Of course, this cannot rule out the accuracy of data statistics.

\subsection{Error Evaluation}

In order to evaluate the forecasting accuracy of the proposed model and measure the error effectively, Sum of Square Error (SSE), Mean Absolute Error (MAE), and Root Mean Square Error (RMSE) are applied as the evaluation criteria in this study. The sizes of these three indicators are able to reflect the accuracy of the prediction, the smaller the size of indicator, the better the prediction performance. The SSE, RMSE, and MAE are defined as:

$$
\begin{aligned}
S S E & =\sum_{t=1}^{T}\left(x_{t}-\hat{x}_{t}\right)^{2} \\
R M S E & =\sqrt{\frac{1}{T} \sum_{t=1}^{T}\left(x_{t}-\hat{x}_{t}\right)^{2}} \\
M A E & =\frac{1}{T} \sum_{t=1}^{T}\left|x_{t}-\hat{x}_{t}\right|
\end{aligned}
$$

where $x(t)$ and $\hat{x}(t)$ represent the real values and forecasting values at time point $t$. And $T$ is the data size of testing set. 
The results are listed in Table 1. Besides, the error indicators of the first 100 prediction values in the test set are also calculated as shown in Table 2.

Table 1. Comparison of forecasting performance of different markets.

\begin{tabular}{cccc}
\hline & SSE & RMSE & MAE \\
\hline EU ETS & 35.9067 & 0.4466 & 0.2899 \\
Shenzhen pilot & 18.3315 & 0.3274 & 0.2442 \\
Hubei pilot & 1.489 & 0.0892 & 0.0663 \\
\hline
\end{tabular}

Table 2. Comparison of forecasting performance of first 100 values.

\begin{tabular}{lcccccc}
\hline & SSE & $(\%)$ & RMSE & $\begin{array}{c}\text { Reduced } \\
\text { proportion } \\
(\%)\end{array}$ & MAE & $\begin{array}{c}\text { Reduced } \\
\text { proportion } \\
(\%)\end{array}$ \\
\hline $\begin{array}{l}\text { EU ETS } \\
\begin{array}{l}\text { Shenzhen } \\
\text { pilot }\end{array}\end{array}$ & 1.9376 & 94.60 & 0.1392 & 68.83 & 0.1076 & 62.88 \\
$\begin{array}{l}\text { Hubei } \\
\text { pilot }\end{array}$ & 0.78549 & 40.84 & 0.3293 & 0.58 & 0.2468 & 1.06 \\
\hline
\end{tabular}

From the results of Table 1, it can be found that, firstly, the EMD-SVM model has a much higher error in the EU ETS on the SSE, RMSE and MAE than these in the two carbon trading pilots of China. Meanwhile, the short-term forecasting performance of the EU ETS is good, and the error fluctuation is small, but SSE, RMSE and MAE measure the overall predictive ability of the model. Secondly, the same model has different prediction performance for different markets. This paper only mines information from historical information to predict carbon prices. However, at some point in different markets, carbon prices contain historical information that is not necessarily equal in length of time, and some information that may be failure to be mined by the model affects the predictions. In terms of overall forecast accuracy, the prediction ability of EMD-SVM in China ETS pilots is superior to that in the EU ETS.

Table 2 is the error indicator of the first 100 predicted values (short-term) in the test set and the comparison with the error of all the test sets. Table 2 dedicates that the SSE, RMSE and MAE of the first 100 predicted values of the EU ETS have a large degree of decline. This fully demonstrates that the short-term forecasting ability of the EMD-SVM model in the EU ETS is far better than that in China ETS pilots.

\section{Conclusion}

The contributions of this paper are:

(1) For the nonlinear and non-stationary characteristics of carbon prices, the EMD-SVM model is used to predict the prices. Firstly, the original carbon prices of multi-ETS are decomposed to obtain different IMFs. The SVM is used to mine historical carbon prices information from IMFs to train the model and predict the future prices.

(2) Using the prices data of multi-ETS, this paper conducts empirical analysis to explore the prediction performance of the EMD-SVM model in different markets. The overall trend of the EMD-SVM model in the three markets is almost the same as the real values, which is consistent with the trend of carbon prices. But the prediction of the specific prices does not reach high accuracy. When predicting carbon prices of China ETS pilots, the short-term forecast of this model has a smaller error and better prediction performance than the longterm forecast. In terms of overall forecast accuracy, EMDSVM is better predicted in China ETS than in the EU ETS.

In this paper, the same prediction model is used for different IMFs, but different IMFs have different frequencies, so the applicability of the model will affect the prediction performance. In future research, we will find suitable models for different IMFs to improve accuracy.

\section{Acknowledgments}

This work was supported by the National Natural Science Foundation of China (No. 71704098, 71971039), the Natural Science Foundation of Shandong Province (No. ZR2016GQ03).

\section{References}

1. H. Ghoddusi, G.G. Creamer, N. Rafizadeh, Energy Econ., 81(2019)

2. T. Bollerslev, Eeri Research Paper, 31, 3(1986)

3. Y. Miyanaga, N. Nagai, N. Miki, IEEE Trans. Circuits Syst., 34, 6(1987)

4. S.J. Byun, H. Cho, Energy Econ., 40(2013)

5. B. Zhu, J. Chevallier, Appl. Econ. Lett., 22, 5(2015)

6. V. Cherkassky, Technometrics, 38, 4(1997)

7. B. Zhu, Y. Wei. System Eng. Theor. Prac., 31, 12(2011)

8. N. E. Huang, Z. Shen, S.R. Long, M.C. Wu, H.H. Shih, Q. Zheng, N. Yen, C. Tung, H. Liu., Proc. R. Soc. London, Ser. A, 454, 1971(1998)

9. X. Gong, F. Wen, C. Huang, X. Yang. Mana. Rev., 29, 1(2017)

10. R. Zhou, R. Chen, Y. Chen, J. Lu, S. Zhou, L. Liu. System Eng. Theor. Prac., 36, 3(2016)

11. Gao Y., Li J., CJPRE, 24, 6(2014)

12. M.S. AI-Musaylh, R.C. Deo, L. Yan, F. Jan, Appl. Energy, 217(2018)

13. C. Cortes, V. Vapnik, Mach. Learn., 20, 3(1995)

14. J. Lago, F.D. Ridder, B.D. Schutter, Appl. Energy, 221(2018)

15. A. Lepone, R.T. Rahman, Y.Y. Jin, LCE, 2, 2(2011)

16. S. Viteva, Y. V. Veld-Merkoulova, K. Campbell, Energy Econ., 45, 6(2014)

17. J. Chevallier, F. Ielpo, L. Mercier, Energy Policy, 37, 1(2009)

18. L.T. Zhao, Y. Wang, S.Q. Guo, G. Zeng, Appl. Energy, 220(2) 